\title{
DETECTION OF CROSSOVER \& BIFURCATION POINTS ON A RETINAL FUNDUS IMAGE BY ANALYZING NEIGHBORHOOD CONNECTIVITY OF NON-VASCULAR REGIONS AROUND A JUNCTION POINT
}

\author{
Harsh Fatepuria', Govind Singhania ${ }^{2}$, Nishit $\mathrm{Shah}^{3}$, Rahul Singh ${ }^{4}$, Nilanjana Dutta Roy \\ ${ }^{1}$ Student, Computer Science \& Engineering Department, IEM, WB, India \\ ${ }^{2}$ Student, Computer Science \& Engineering Department, IEM, WB, India \\ ${ }^{3}$ Student, Computer Science \& Engineering Department, IEM, WB, India \\ ${ }^{4}$ Student, Computer Science \& Engineering Department, IEM, WB, India \\ ${ }^{5}$ Assistant Professor, Computer Science \& Engineering Department, IEM, WB, India
}

\begin{abstract}
Over the last few decades, analysis of retinal vascular images has gained its popularity among the cyber scientists. Retina is unique in nature because of its most important feature, bifurcation \& crossover points, serving reliable authentication basis. Using these two points many problem of authentication could be handled easily. Literature has shown that in a retinal vascular structure, bifurcation\& crossover points need to be separated for the purpose of authentication. With this motivation in mind, we propose a novel method to segregate vascular bifurcation points from crossover points in any retinal image by analyzing neighborhood connectivity of nonvascular regions around a junction point on retinal blood vessels.
\end{abstract}

Keywords: Retinal vessel analysis, retinal blood vessels, bifurcation and crossover point's detection.

\section{INTRODUCTION}

With the immense growth of digital automation, the demand for the automated computer-aided analysis of medical images has been emerging as a potential research domain. The multifarious growth of Internet technology in modern cyber age has been producing alarming threat to the traditional security system. To ensure better security, cyber scientists are searching for biometric features as the basis of authentication. The motive behind using the biological features is its uniqueness in nature. Retina is one of the most secure, reliable, trustworthy sources of biometric authentication. Retina is unique because of its anatomical structure of retinal nerves. One of the unique features of retina is the bifurcation point [7]. It is basically a junction on a vessel from where two other child nerves are generated [Fig. 2]. The proposed algorithm focuses on determining the potential bifurcation and crossover points from the segmented binary fundus images of retina collected from DRIVE database [5]. The analysis of geometrical properties of branching structure is not new but retinal blood vessel tree geometry and topology has not been widely studied by means of image processing. Perez et al. [3] proposed a geometric feature based technique for detecting vascular bifurcation and crossover points (landmarks) based on skeletonized vascular network. They characterize the bifurcation and crossover points by the total number of intersections using a fixed-size circular window centered on the candidate landmark points. This process extracts the morphological features to identify the landmarks of retinal vascular tree. However, it fails when two true bifurcation points are very close window. This process is also prone to false identifying landmarks if another vessel passes through the fixed-size window. Jung et al. [2] proposed cross perpendicular structure and Y-type structure with eight connected neighbors for vascular landmarks extraction. Bevilacqua et al. [1] and Chanwimaluang et al. [4] also proposed similar techniques to detect landmarks based on some specific formation of vessel centre-line pixels analyzing their eight- connectivity nature. But all these methods tend to detect landmarks wrongly due to the error induced by skeletonization because of abrupt change of the width of any vessel within the mask window. In this paper, a novel but simple approach is proposed to detect the vessel bifurcation and crossover points analyzing the intersection/junction regions on the blood vessels. Next section introduces the proposed method of vessel bifurcation along with brief background knowledge of retinal image analysis. The rest of the paper is organized as follows. The detailed illustration of the proposed algorithm is provided in section 2. Section 3 provides the experimental results, after which the conclusion is drawn followed by references. 


\section{PROPOSED SCHEME}

One of the unique features of any human retina is its Bifurcation \& Crossover points. Bifurcation is basically a junction (Fig. 2) on a vessel from where two other child nerves are generated. On the other hand, when two vessels or branches of two vessels meet at a point then it is termed as Crossover (Fig. 1). Statistical analysis has shown that in a retinal vascular structure, bifurcation \& crossover points are the most important features used for many purposes. Therefore the proposed algorithm has been attempted for pointing out the prominent bifurcation \& crossover points on the retinal blood vessels. The overall scheme is outlined below.

The scheme takes vessel segmented binary image as input (Fig. 4). The vessels can be extracted from the fundus image (Fig 3) by various image filtering techniques as discussed in $[2,4]$.

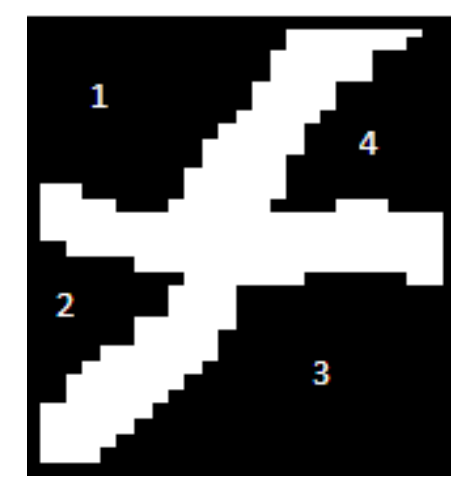

Fig -1: A window detected as crossover because of four black regions.

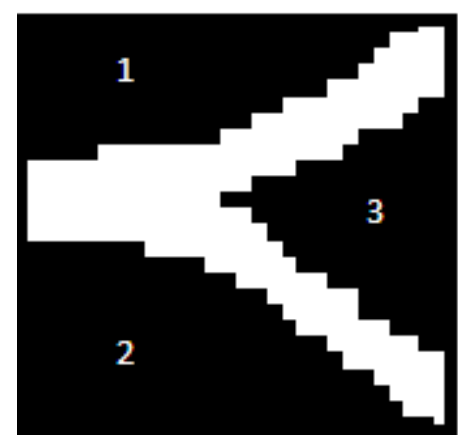

Fig -2: A window detected as bifurcation because of three black regions.

Start traversing the border pixels of the image from the top left corner in anticlockwise way. Mark the first black pixel as region 1. Continue to traverse the image until there is a transformation from white pixel to black pixel. Apply Breadth First Search algorithm to check whether the new black pixel meets any other previously found black region. If it does not meet, increment the region number, otherwise not. Similarly, traverse all the border pixels and keep counting the number of total black regions. If number of black regions is equal to three, it is a bifurcation (Fig 2); and if the number of black regions exceeds 3 , then it is crossover (Fig 1).

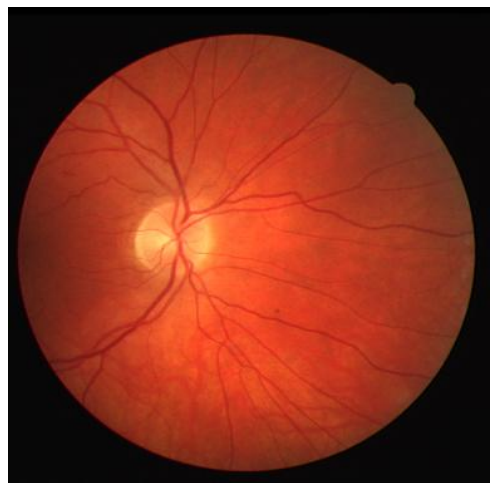

Fig -3 Image from DRIVE database

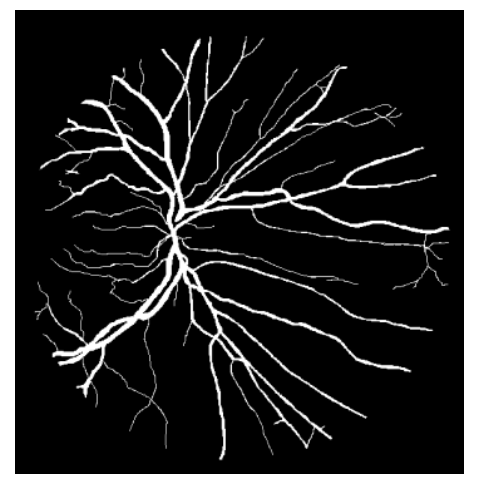

Fig -4 Vessel segmented image from DRIVE database

\section{PROPOSED ALGORITHM}

Input: Vessel Segmented Binary Fundus Image (fig 4) Output: Image showing bifurcation \& crossover points

Step 1 Consider a window with maximum number of white pixels as the root.

Step 2 A square window is traversed throughout the image row wise to count the number of black regions in the window. Step 2.1 To count the black regions, initialize a variable (count) as 1 .

Step 2.2 If the first (top left) pixel is black, insert its location in an array with region number 1 and increment count variable.

Step 2.3 Traverse the border pixels of the box in anticlockwise direction.

Step 2.4 On detecting a change in color from white to black (let the black pixel be called BP), follow Step 2.4.1 otherwise continue traversing the border pixels.

Step 2.4.1 If the count variable equals 2 , insert the black pixel's (i.e., BP's) locations in the previous mentioned array 
with region number 2 and increment count; otherwise using BFS (Breadth First Search) technique add all the neighboring (i.e., top, bottom, left and right) black pixels of BP in a queue to see whether it meets any black pixel already present in the previous array.

Step 2.4.2 If it does not meet any black pixel present in the array, insert BP's locations in the array with the count value as region number; and increment the count variable; otherwise continue traversing the border pixels.

Step 2.5 Return the number of black regions in the box as (count-1).

Step 3 If the number of black regions is less than or equal to 2, ignore the box.

Step 4 If the number of black regions is equal to 3 , color the region red, indicating bifurcation.

Step 5 If the number of black regions is greater than 3, color the region blue, indicating crossover.

\section{EXPERIMENTAL RESULTS}

- Crossover Points

- Bifurcation Points

\subsection{Results on cropped images taken from Drive}

Database Showing Crossover \& Bifurcation Points

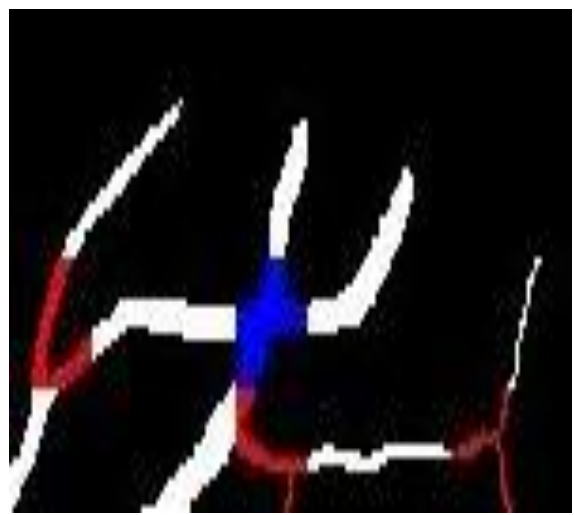

Fig -5

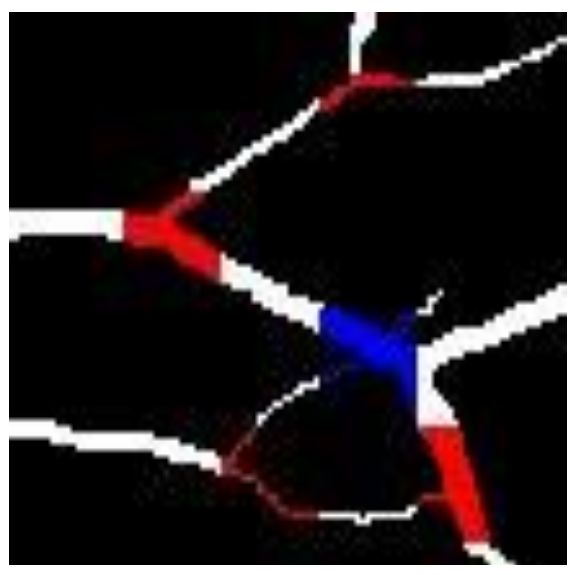

Fig -6

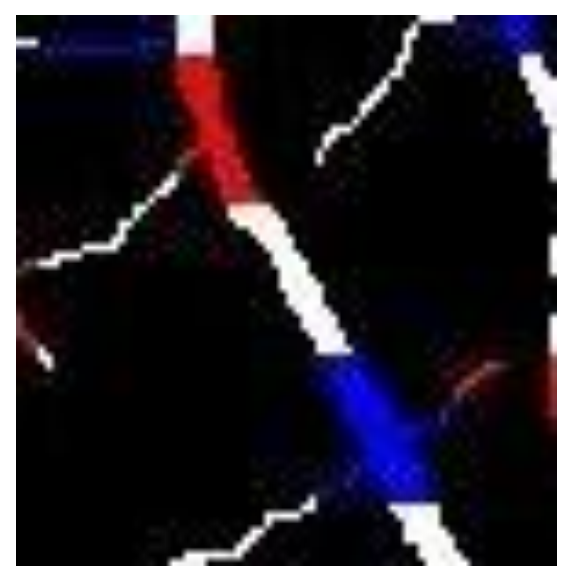

Fig -7

\subsection{Results on Fundus Images taken from Drive} Database

Table-1: Results

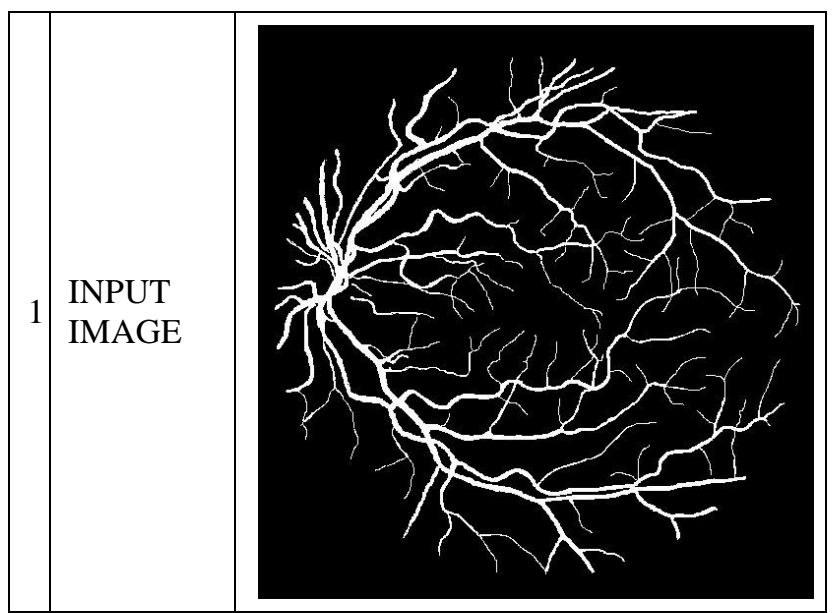



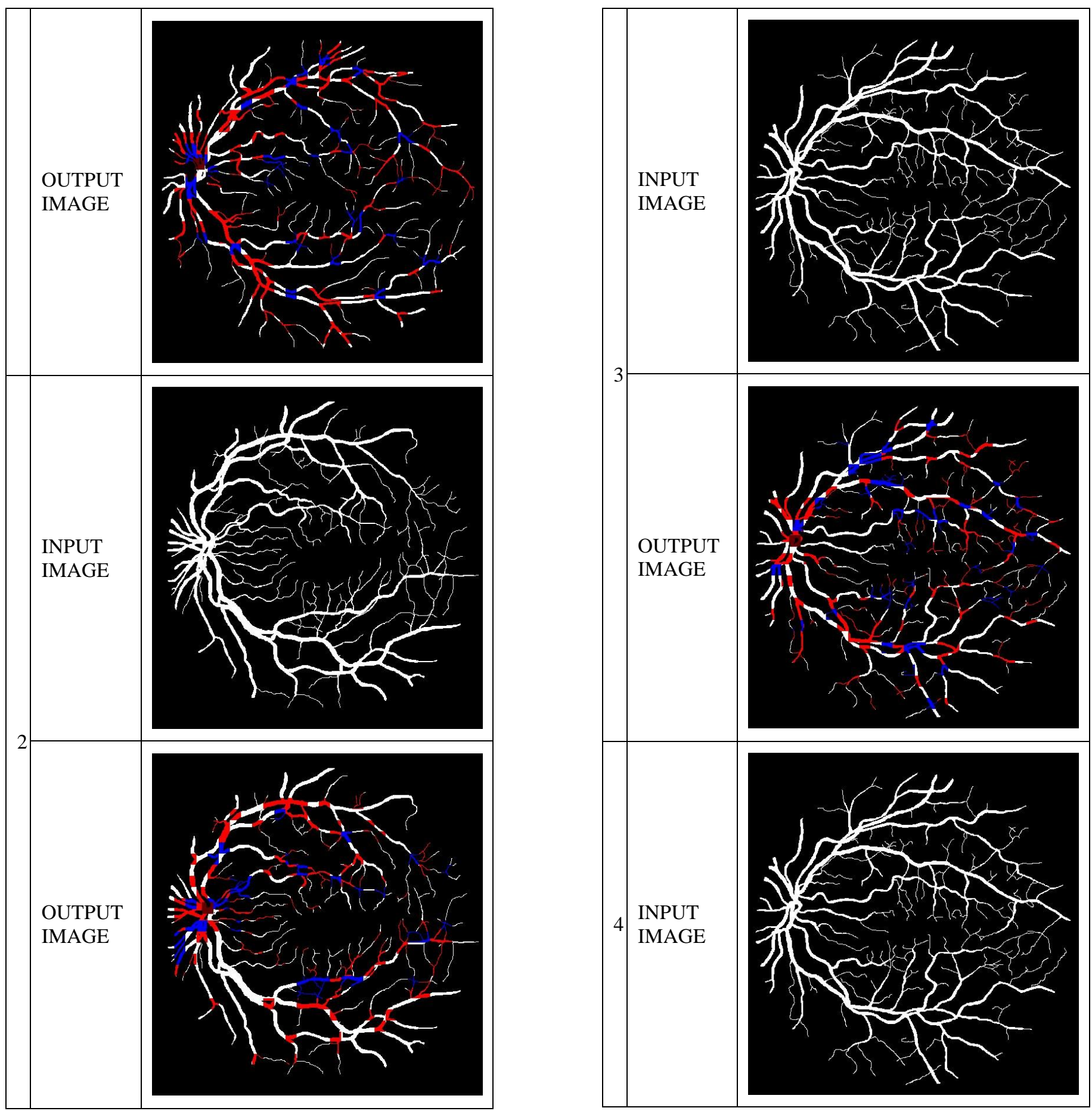

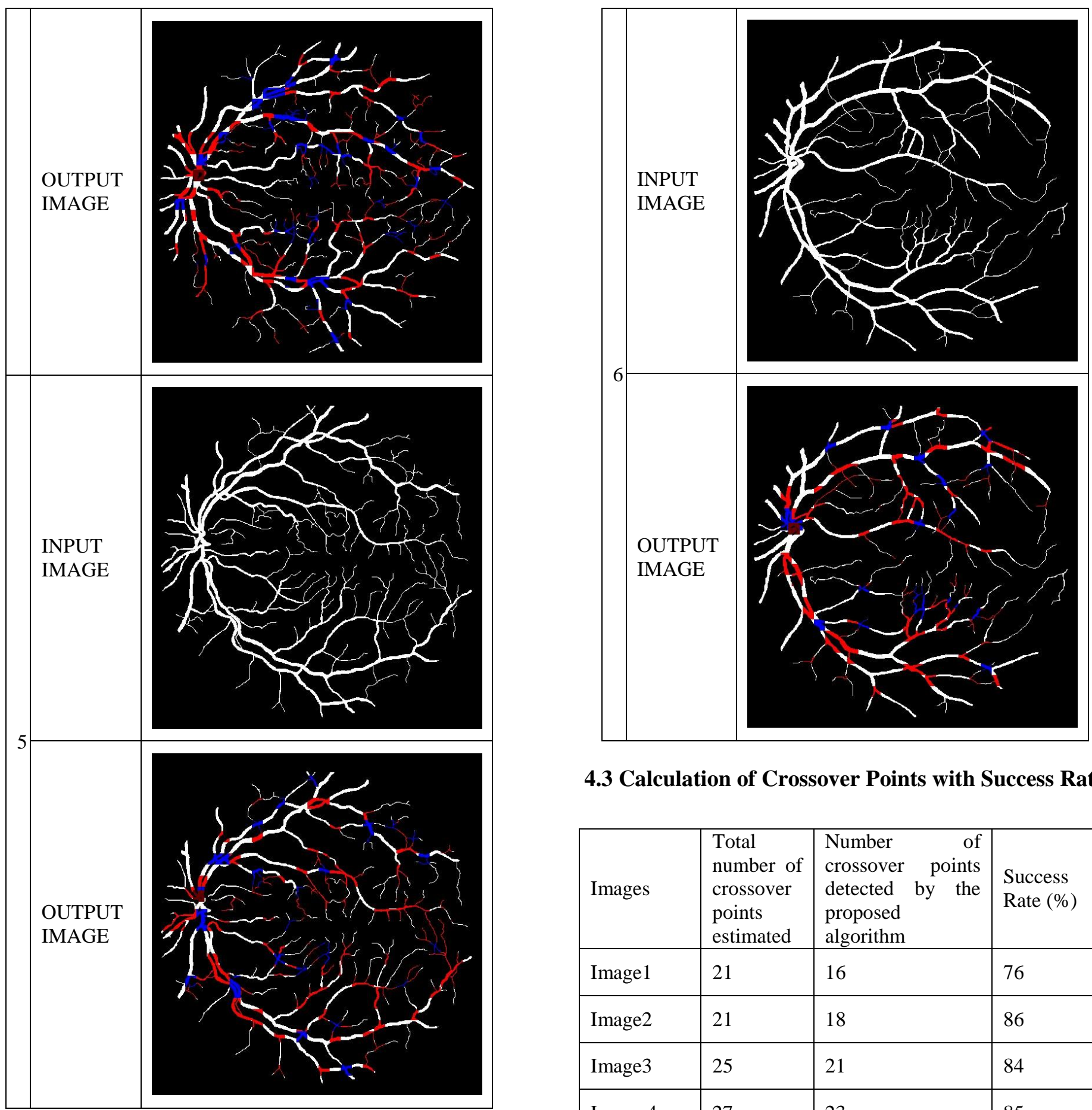

4.3 Calculation of Crossover Points with Success Rate

\begin{tabular}{|l|l|l|l|}
\hline Images & $\begin{array}{l}\text { Total } \\
\text { number of } \\
\text { crossover } \\
\text { points } \\
\text { estimated }\end{array}$ & $\begin{array}{l}\text { Number } \\
\text { crossover points } \\
\text { detected by the } \\
\text { proposed } \\
\text { algorithm }\end{array}$ & $\begin{array}{l}\text { Success } \\
\text { Rate (\%) }\end{array}$ \\
\hline Image1 & 21 & 16 & 76 \\
\hline Image2 & 21 & 18 & 86 \\
\hline Image3 & 25 & 21 & 84 \\
\hline Image 4 & 27 & 23 & 85 \\
\hline Image 5 & 24 & 22 & 92 \\
\hline Image 6 & 20 & 14 & 70 \\
\hline Average Success Rate & & 82 \\
\hline
\end{tabular}




\section{CONCLUSIONS}

In this paper, a novel method is proposed to separate vascular bifurcation points from its crossover points in any retinal images by analyzing neighborhood connectivity of nonvascular regions around a junction point on retinal blood vessels. Statistical analysis has shown that the result of this scheme is effective in separating the prominent bifurcation points from crossover points with reasonable accuracy and time. By using this concept, in near future, preparing the authentication-based schemes would be much easier.

The results are generated in $\mathrm{C}$ language using gcc compiler and it is tested on Intel i3 processor with 4 GB RAM

\section{REFERENCES}

[1]. V. Bevilacqua, S. Cambo, L. Cariello, and G. Mastronardi, ìa combined method to detect retinal fundusfeatures, $\hat{i}$ Proceedings of European Conference on Emergent Aspects in Clinical Data Analysis, pp. 1ñ6, 2005.

[2]. Eunhwa Jung and Kyungho Hong, ìAutomatic retinal vasculature structure tracing and vascular landmark extraction from human eye image, î Proceedings of IEEE International Conference on Hybrid Information Technology, vol. 12, pp. 161 ñ167, 2006.

[3]. M. E. Martinez-Perez, A. D. Highes, A. V. Stanton, S.A.Thorn, N. Chapman, and A. A. Bharath K. H. Parker,ìRetinal vascular tree International Journal of Latest Research in Science and Technology.

ISSN: 2278-5299 108 morphology: a semi-automatic quantification,î IEEE Transactions on Biomedical Engineering, vol. 49(8), 2002.

[4]. T Chanwimaluang and Guoliang Fan, ìAn efficient blood vessel detection algorithm for retinal images using local entropy thresholding ,î Proceedings of the 2003 International Symposium on Circuits and Systems, vol. 5, 2003.

[5]. The DRIVE database, ilmage sciences institute, university medical center utrecht,î The Netherlands. http://www.isi.uu.nl/Research/Databases/DRIVE/, last accessed on 7th July 2007

[6]. W. K. Pratt, Digital Image Processing, Prentice Hall, second edition, 1991. Milan Sonka, Vaclav Hlavac, and Roger Boyle, Image Processing, Analysis and Machine Vision, PWS Publishing, second edition, 1999.

[7]. Automatic detection of bifurcation points in retinal fundus-images 1Sourav Saha, 2Nilanjana Dutta Roy, International Journal of Latest Research in Science and Technology ISSN (Online): 2278-5299 Volume 2,Issue 2: Page No.105-108, March - April (2013) http://www.mnkjournals.com/ijlrst.htm

[8]. Model-Based Method for Improving the Accuracy And Repeatability of Estimating Vascular Bifurcations And Crossovers From Retinal Fundus Images Chia-Ling Tsai, Member, IEEE, Charles V. Stewart, Member, IEEE, Howard L. Tanenbaum, and Badrinath Roysam, Member, IEEE 